\title{
Variabilidade interobservador no diagnóstico da doença trofoblástica gestacional: um estudo na residência de patologia da Universidade Federal do Ceará
}

\section{Interobserver variability in the diagnosis of gestational trophoblastic disease: a study in the pathology residency of the Federal University of Ceará}

\author{
Edson de Macêdo Sousa ${ }^{1,2}$. Diane Isabelle Magno Cavalcante ${ }^{2}$. Lis Caetano Nóbrega Costa Araújo². \\ 1 Hospital Universitário Walter Cantídio (HUWC), Fortaleza, Ceará, Brasil. 2 Universidade Federal do Ceará (UFC), Fortaleza, \\ Ceará, Brasil.
}

\section{RESUMO}

Objetivo: Analisar a variabilidade interobservador no diagnóstico histopatológico de doença trofoblástica gestacional (DTG). Metodologia: Foram selecionados aleatoriamente 50 casos de DTG do arquivo do Departamento de Patologia da Universidade Federal do Ceará, classificados no laudo original como: 31 molas completas, 15 molas parciais e 4 indeterminados. Uma lâmina de cada caso foi analisada por um médico residente e um patologista ginecológico, cujo diagnóstico foi considerado como referência. O laudo original também foi utilizado para comparação. Valor de beta-HCG, quando informado, foi correlacionado com o tipo de mola. Os valores de kappa foram calculados para a concordância interobservador. Resultados: A concordância geral interobservador foi fraca $(\mathrm{K}=0,25)$, entre o residente $\mathrm{x}$ patologista ginecológico e residente $\mathrm{x}$ laudo original o kappa foi respectivamente $\mathrm{K}=0,16$ e $\mathrm{K}=0,38$. A concordância foi moderada $(\mathrm{K}=0,41)$ entre o patologista ginecológico e o laudo original. A dosagem de beta-HCG $>100.000 \mathrm{mUI} / \mathrm{ml}$ ocorreu em 7 molas completas e 4 parciais. Conclusão: A concordância interobservador para o diagnóstico de DTG variou de pobre a moderada quando somente a histologia foi utilizada como ferramenta diagnóstica. Valor alto de beta-HCG foi mais frequente na mola completa.

Palavras-chave: Doença trofoblástica gestacional. Mola hidatiforme. Variações dependentes do observador.

\begin{abstract}
Objective: To analyze interobserver variability in the histopathological diagnosis of gestational trophoblastic disease (GTD). Methodology: Fifty cases of GTD were randomly selected from the Department of Pathology of the Federal University of Ceará, classified in the original report as 31 complete moles, 15 partial moles and 4 non classified. A single slide from each case was evaluated by a resident physician and a gynecological pathologist, which diagnosis was considered as a reference. Original report was applied for correlation as well. Beta-HCG values was correlated with mole type, when provided. Kappa values were calculated for interobserver variability. Results: Overall interobserver agreement was poor $(\mathrm{K}=0.25)$, between resident $\mathrm{x}$ gynecological pathologist and resident $\mathrm{x}$ original report the kappa values were respectively $\mathrm{K}=0.16$ and $\mathrm{K}=0.38$. Concordance was moderate $(\mathrm{K}=$ 0.41 ) even between the gynecological pathologist and the original report. Beta-HCG values higher than $100.000 \mathrm{mIU} / \mathrm{ml}$ were seen in 7 complete and 4 partial moles. Conclusion: Interobserver agreement for the diagnosis of GTD ranged from poor to moderate when histology was the only diagnostic tool. High beta-HCG value was more frequent in complete moles.
\end{abstract}

Keywords: Gestational trophoblastic disease. Hydatidiform mole. Variations dependent on the observer.

Autor correspondente: Edson de Macêdo Sousa, Rua Braz de Francesco, 135, Presidente Kennedy, Fortaleza, Ceará. CEP: 60355-633. Telefone: +55 85 99629-4013. E-mail: macedo.sb@hotmail.com

Conflito de interesses: Não há qualquer conflito de interesses por parte de qualquer um dos autores.

Recebido em: 30 Jul 2018; Revisado em: 08 Dez 2018; Aceito em: 08 Dez 2018. 


\section{INTRODUÇÃO}

A doença trofoblástica gestacional (DTG) constitui um espectro de patologias caracterizadas pela proliferação anormal do trofoblasto. As lesões incluem mola hidatiforme completa e parcial, mola invasiva, coriocarcinoma e o tumor trofoblástico do sítio placentário ${ }^{1}$. Sua forma mais comum, mola hidatiforme, ocorre em torno de 1:200-400 gestações no Brasil, cerca de 5 a 10 vezes mais frequente que na Europa e América do Norte. Os extremos de idade e história obstétrica pregressa de DTG são fatores de risco para essa patologia ${ }^{2}$.

A mola hidatiforme completa (MHC) resulta da fecundação de um óvulo com núcleo ausente ou inativo por um espermatozoide ou mais raramente por dois espermatozoides, originando uma célula diploide. Não há elementos fetais. Já o cariótipo da mola hidatiforme parcial (MHP) é triploide, resultado da fertilização de um óvulo normal por dois espermatozoides ou um espermatozoide diploide. Pode haver feto, porém é inviável e apresenta crescimento intrauterino restrito e múltiplas malformações ${ }^{3}$. Ao exame histológico, as MHC exibem anormalidades que envolvem a totalidade ou a maior parte do tecido viloso. As vilosidades coriônicas estão aumentadas, apresentando forma côncava com cavitação central e não possuem vasos adequadamente desenvolvidos. Entretanto, a característica mais marcante é a extensiva proliferação trofoblástica, que envolve toda a circunferência dos vilos. Em contraste, as molas parciais demostram aumento dos vilos e perturbação da arquitetura em apenas uma parte deles. A proliferação trofoblástica é moderada e focal $^{4}$.

Os níveis de beta-HCG entre as portadoras de MHC são bastante elevados e quase metade das pacientes têm níveis superiores a $100.000 \mathrm{mUI} / \mathrm{mL}$. No entanto, os níveis são bem menores entre os casos com MHP e valores superiores a $100.000 \mathrm{mUI} / \mathrm{mL}$ são observados em apenas $10 \%$ dos casos $^{5}$.

O diagnóstico adequado do tipo de mola é muito importante para o seguimento clínico, pois determina o risco de doença molar persistente que é mais alto na MHC. Quando as características morfológicas das molas estão bem desenvolvidas, o diagnóstico histológico não oferece dificuldades, entretanto estudos têm demonstrado que o diagnóstico morfológico das molas, especialmente nas suas formas mais precoces, sofre de uma baixa reprodutibilidade interobservador ${ }^{6}$.

O exame histopatológico continua sendo o padrão ouro diagnóstico e atualmente pode-se utilizar como complementação diagnóstica nos casos dúbios entre mola parcial e completa a imunocoloração para p57, um inibidor do ciclo celular. O gene p57KIP2 é transcrito pela mãe, mas o imprinting é determinado pelo pai, e apresenta expressão no tecido decidual materno, como no citotrofoblasto e em células estromais das vilosidades, quando o material genético estiver presente no concepto, como ocorre na MHP. Em contraste, uma vez que os dois cromossomos x das MHC são derivados do pai, não ocorre expressão da proteína p57 no citotrofoblasto ou em células estromais dos vilos dessas molas $^{3}$. A implementação dessa imunocoloração associado a padronização de critérios morfológicos, informação do valor do beta-HCG e a ploidia definida pela citometria de fluxo contribuiriam para reduzir a variabilidade diagnóstica interobservador.

O objetivo desse estudo é analisar a variabilidade interobservador no diagnóstico histopatológico de doença trofoblástica gestacional (DTG) no serviço de patologia da Universidade Federal do Ceara no contexto da residência médica.

\section{MATERIAL E MÉTODOS}

Pesquisa observacional, do tipo coorte transversal, utilizando 50 casos selecionados de forma aleatória dentre 212 casos de DTG provenientes da Maternidade Escola Assis Chauteaubriand (MEAC) no período de 2012 a 2015 e que foram diagnosticados por patologista ginecológico no Departamento de Patologia da UFC. O diagnóstico do patologista ginecológico foi considerado como referência. Os casos foram codificados por números e vistos de forma cega e independente por um médico residente de anatomia patológica e por um patologista ginecológico. Os casos foram classificados em MHC, MHP, aborto hidrópico $(\mathrm{AH})$, aborto usual (A) e indeterminados de acordo com critérios histopatológicos clássicos descritos na literatura especializada. $\mathrm{O}$ valor do beta-HCG , informado na solicitação do exame histopatológico, foi comparado com o tipo de DTG. Os dados foram analisados para concordância percentual e teste Kappa. O índice de Kappa (к) menor que 0,4 indica fraca concordância, entre 0,4 e 0,75 moderada concordância e maior que 0,75 excelente concordância. $\mathrm{O}$ projeto foi aprovado pelo comitê de ética em pesquisa da Maternidade Escola Assis Chauteaubriand (MEAC), parecer $\mathrm{N}^{\circ} 103.998$.

\section{RESULTADOS}

A idade das pacientes variou de 15 a 48 anos. Os laudos histopatológicos originais dos 50 casos selecionados foram de 31 MHC, 15 MHP e 4 indeterminados. A concordância geral interobservador foi $32 \%(\mathrm{~K}=0,25)$, em 11 casos de MHC, 4 de MHP e 1 indeterminado. Entre o residente e diagnóstico do patologista foi de $36 \%(\mathrm{~K}=0,16)$, em 11 casos de MHC, 5 de MHP e 2 indeterminados. Entre residente e o laudo original foi de $60 \%(\mathrm{~K}=0,38)$, em 12 casos de MHC, 15 de MHP e 3 indeterminados. Já a concordância entre patologista ginecológico e o laudo original foi $64 \%(\mathrm{~K}=0,41)$, em 27 casos de MHC, 4 de MHP e 1 indeterminado (Tabela 1). A dosagem de beta-HCG foi informada na solicitação médica em 46 casos e foi maior que $100.000 \mathrm{mUI} / \mathrm{ml}$ em 11 pacientes $(7$ molas completas e 4 parcial). 
Tabela 1. Concordância no diagnóstico histológico.

\begin{tabular}{|c|c|c|c|c|c|}
\hline Casos & Idade & HCG (mIU/ml) & Laudo & Patologista & Residente \\
\hline 1 & 37 & 609,4 & $\mathrm{C}$ & $\mathrm{C}$ & $\mathrm{P}$ \\
\hline 2 & 43 & $<1,2$ & I & $\mathrm{C}$ & $\mathrm{P}$ \\
\hline 3 & 15 & 507 & $\mathrm{C}$ & $\mathrm{C}$ & $\mathrm{P}$ \\
\hline 4 & 25 & 546.699 & $\mathrm{P}$ & $\mathrm{AH}$ & $\mathrm{P}$ \\
\hline 5 & 38 & 47.253 & $\mathrm{C}$ & $\mathrm{C}$ & $\mathrm{P}$ \\
\hline 6 & 39 & $>200.000$ & $\mathrm{C}$ & $\mathrm{C}$ & $\mathrm{P}$ \\
\hline 7 & 29 & 703,5 & $\mathrm{C}$ & $\mathrm{C}$ & $\mathrm{C}$ \\
\hline 8 & 24 & $>200.000$ & $\mathrm{C}$ & $\mathrm{C}$ & $\mathrm{C}$ \\
\hline 9 & 46 & 4318 & $\mathrm{P}$ & $\mathrm{P}$ & $\mathrm{P}$ \\
\hline 10 & 25 & $>200.000$ & $\mathrm{C}$ & $\mathrm{C}$ & $\mathrm{P}$ \\
\hline 11 & 19 & $\operatorname{xxxxx}$ & I & I & I \\
\hline 12 & 29 & 2007 & $\mathrm{C}$ & $\mathrm{P}$ & $\mathrm{P}$ \\
\hline 13 & 19 & 18 & $\mathrm{P}$ & $\mathrm{P}$ & $\mathrm{P}$ \\
\hline 14 & 21 & 10.313 & $\mathrm{C}$ & I & $\mathrm{P}$ \\
\hline 15 & 19 & 54930 & $\mathrm{P}$ & $\mathrm{AH}$ & $\mathrm{P}$ \\
\hline 16 & 37 & 155.447 & $\mathrm{C}$ & $\mathrm{C}$ & $\mathrm{C}$ \\
\hline 17 & 48 & 7.958 & $\mathrm{P}$ & $\mathrm{C}$ & $\mathrm{P}$ \\
\hline 18 & 20 & 1.000 & $\mathrm{C}$ & $\mathrm{C}$ & $\mathrm{P}$ \\
\hline 19 & 39 & 1.007 & $\mathrm{C}$ & $\mathrm{C}$ & $\mathrm{C}$ \\
\hline 20 & 16 & 95.422 & $\mathrm{C}$ & $\mathrm{C}$ & $\mathrm{P}$ \\
\hline 21 & 42 & $<1$ & $\mathrm{P}$ & A & $\mathrm{P}$ \\
\hline 22 & 30 & 974 & $\mathrm{C}$ & $\mathrm{C}$ & $\mathrm{C}$ \\
\hline 23 & 26 & $>255.000$ & $\mathrm{C}$ & $\mathrm{C}$ & $\mathrm{P}$ \\
\hline 24 & 25 & xxxxx & $\mathrm{P}$ & $\mathrm{C}$ & $\mathrm{P}$ \\
\hline 25 & 16 & 4925 & $\mathrm{C}$ & $\mathrm{C}$ & $\mathrm{C}$ \\
\hline 26 & 18 & $>200.000$ & $\mathrm{C}$ & $\mathrm{C}$ & $\mathrm{P}$ \\
\hline 27 & 32 & $>200.000$ & $\mathrm{C}$ & $\mathrm{C}$ & $\mathrm{C}$ \\
\hline 28 & 21 & 335,4 & $\mathrm{P}$ & $\mathrm{C}$ & $\mathrm{P}$ \\
\hline 29 & 27 & $<1$ & $\mathrm{P}$ & $\mathrm{P}$ & $\mathrm{P}$ \\
\hline 30 & 24 & 6,3 & I & A & I \\
\hline 31 & 24 & 17733,2 & $\mathrm{C}$ & $\mathrm{C}$ & $\mathrm{P}$ \\
\hline 32 & 41 & 37.320 & $\mathrm{C}$ & I & $\mathrm{C}$ \\
\hline 33 & 21 & 190 & $\mathrm{C}$ & $\mathrm{C}$ & $\mathrm{P}$ \\
\hline 34 & 22 & 3791,8 & $\mathrm{C}$ & $\mathrm{C}$ & $\mathrm{P}$ \\
\hline 35 & 30 & $\operatorname{xxxxx}$ & $\mathrm{P}$ & I & $\mathrm{P}$ \\
\hline 36 & 42 & 126,498 & $\mathrm{P}$ & $\mathrm{C}$ & $\mathrm{P}$ \\
\hline 37 & 19 & 682 & $\mathrm{C}$ & $\mathrm{C}$ & $\mathrm{C}$ \\
\hline 38 & 16 & 90809 & $\mathrm{C}$ & $\mathrm{C}$ & $\mathrm{C}$ \\
\hline 39 & 18 & $<0,1$ & $\mathrm{C}$ & $\mathrm{C}$ & $\mathrm{C}$ \\
\hline 40 & 35 & 33,5 & $\mathrm{C}$ & I & I \\
\hline 41 & 19 & 960,6 & $\mathrm{C}$ & $\mathrm{C}$ & $\mathrm{P}$ \\
\hline 42 & 24 & 19.072 & $\mathrm{C}$ & $\mathrm{C}$ & $\mathrm{P}$ \\
\hline 43 & 26 & $\mathrm{xxxxx}$ & I & A & $\mathrm{I}$ \\
\hline 44 & 32 & $>270.000$ & $\mathrm{P}$ & $\mathrm{P}$ & $\mathrm{P}$ \\
\hline 45 & 17 & 138.583 & $\mathrm{P}$ & $\mathrm{C}$ & $\mathrm{P}$ \\
\hline 46 & 16 & 48.995 & $\mathrm{C}$ & $\mathrm{C}$ & $\mathrm{P}$ \\
\hline 47 & 48 & 95 & $\mathrm{P}$ & $\mathrm{C}$ & $\mathrm{P}$ \\
\hline 48 & 25 & $<1$ & $\mathrm{P}$ & $\mathrm{C}$ & $\mathrm{P}$ \\
\hline 49 & 27 & $<0,1$ & $\mathrm{C}$ & $\mathrm{C}$ & $\mathrm{P}$ \\
\hline 50 & 18 & $<2$ & $\mathrm{C}$ & $\mathrm{C}$ & $\mathrm{C}$ \\
\hline
\end{tabular}

C: Mola Completa; P: Mola Parcial; I: Mola Indeterminada; A: Aborto; AH: Aborto Hidrópico. 


\section{DISCUSSÃO}

Patologistas em sua rotina geralmente se deparam com produtos de abortamento representados na histologia por vilosidades coriônicas hidrópicas e tem que definir o diagnóstico. O nosso índice de concordância diagnóstica geral foi fraco $(\mathrm{k}$ $=0.25$ ) ocorrendo em $32 \%$ do total de 50 casos (Tabela 1). Estudo semelhante relata concordância intraobservador com 5 patologistas em $60 \%$ do total de 50 casos usando apenas critérios morfológicos padronizados de Szulman e Surti para definir o diagnóstico; ou seja, o diagnóstico MHC é feito quando há edema de todas as vilosidades com formação de cisternas centrais, ausência de embrião e hiperplasia trofoblástica circunferencial (Figura 1). O diagnóstico de MHP ocorre quando há envolvimento parcial das vilosidades (normais e hidrópicas), presença de feto, hiperplasia trofoblástica focal leve a moderada e inclusões trofoblásticas (Figura 2). Nesse mesmo estudo também foi avaliado a concordância intraobservador, com resultado variando entre $50 \%$ a $90 \%$ de concordância ${ }^{7}$.

Figura 1. Microfotografia de vilosidade hidrópica, com cisterna central e hiperplasia do trofoblasto diagnosticada pelos observadores como MHC (caso 8; HE 100x).

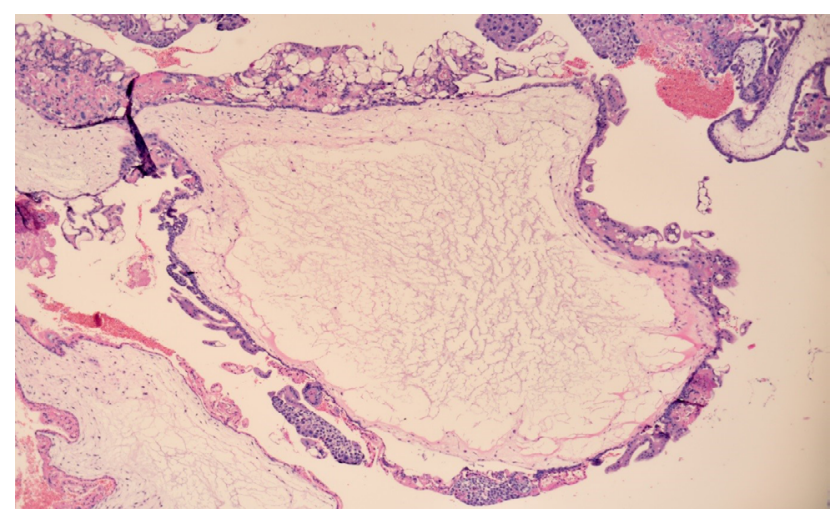

Figura 2. Vilosidades coriônicas com hiperplasia trofoblástica leve e focal. Os três observadores diagnosticaram como MHP (caso 32; HE 100x).

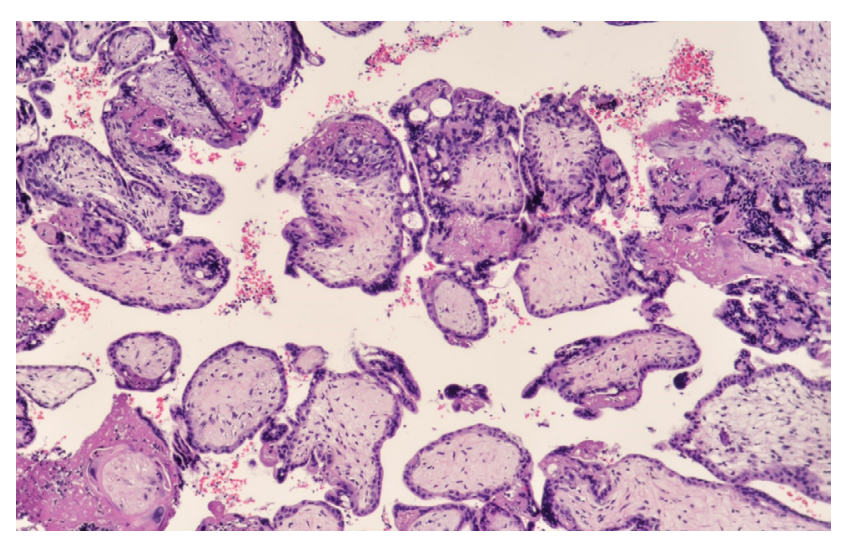

Existem alguns motivos para essa baixa concordância em nosso estudo. Em primeiro lugar todos os casos selecionados eram de gestações do primeiro trimestre estando os vilos pouco desenvolvidos e os critérios descritos em livros didáticos são derivados de estudos em molas de idade gestacional mais avançada. Segundo a hiperplasia trofoblástica na MHP é focal e leve, que torna a identificação difícil. Exemplo é a proliferação trofoblástica polar, própria do $\mathrm{AH}$ que pode ser confundido como critério de MHP. Em terceiro lugar foi utilizada somente uma lâmina por caso e a literatura mostra que as vezes é necessário a avaliação de até dez blocos para confirmar uma hiperplasia trofoblástica focal em uma MHP. Informações clínicas de níveis séricos de beta-HCG, exames de imagem e uma boa descrição macroscópica são de fundamental importância na complementação diagnóstica, pois níveis de beta-HCG entre as portadoras de MHC são bastante elevados e quase metade das pacientes têm níveis superiores a 100.000 $\mathrm{mUI} / \mathrm{mL}$. No entanto, os níveis são bem menores entre os casos com MHP e valores superiores a $100.000 \mathrm{mUI} / \mathrm{mL}$ são observados em apenas $10 \%$ dos casos $^{5}$. Nosso levantamento constatou dosagem de beta $\mathrm{HCG}>100.000 \mathrm{mUI} / \mathrm{ml}$ em 11 pacientes (7 molas completas e 4 parcial), porém essa informação não estava presente no momento do diagnóstico. A descrição macroscópica do produto histopatológico também não foi utilizada como critério pelos observadores desse estudo.

O exame histopatológico continua sendo o padrão ouro diagnóstico, porém em casos dúbios entre MHP, MHC e $\mathrm{AH}$ faz-se necessário utilizar métodos complementares. $\mathrm{O}$ diagnóstico entre MHC e MHP é relativamente simples, porém em gestações em estados iniciais como em nosso relato suscitam discordância diagnóstica, como mostrado na Figura 3. O estudo imuno-histoquímico com p57 estaria indicado, pois seria positivo nas MHP devido à presença de material genético materno e negativo nas $\mathrm{MHC}$ devido à origem essencialmente androgenética. A citometria de fluxo para determinar a ploidia do DNA também seria útil, pois evidenciaria diploidia nas MHC e triploidia nas MHP. Em estudo semelhante da avaliação interobservador de 38 casos de mola hidatiforme, apresentou concordância entre diagnóstico histológico e imunocoloração para p57 em 90,9 \% das MHC e 100\% das MHP. Mostrou ainda concordância entre diagnóstico histológico e ploidia em $100 \%$ das $\mathrm{MHC}$ e $75 \%$ das $\mathrm{MHP}^{8}$. Outro estudo relatou melhora significativa na concordância entre os patologistas após adição de dados citométricos de fluxo, resultando em concordância interobservador, passando de $60 \%$ para $78 \%{ }^{7}$.

Figura 3. Algumas vilosidades edemaciadas com hiperplasia trofoblástica moderada e focal. Dois observadores diagnosticaram como MHP e um como MHC (HE 100x).

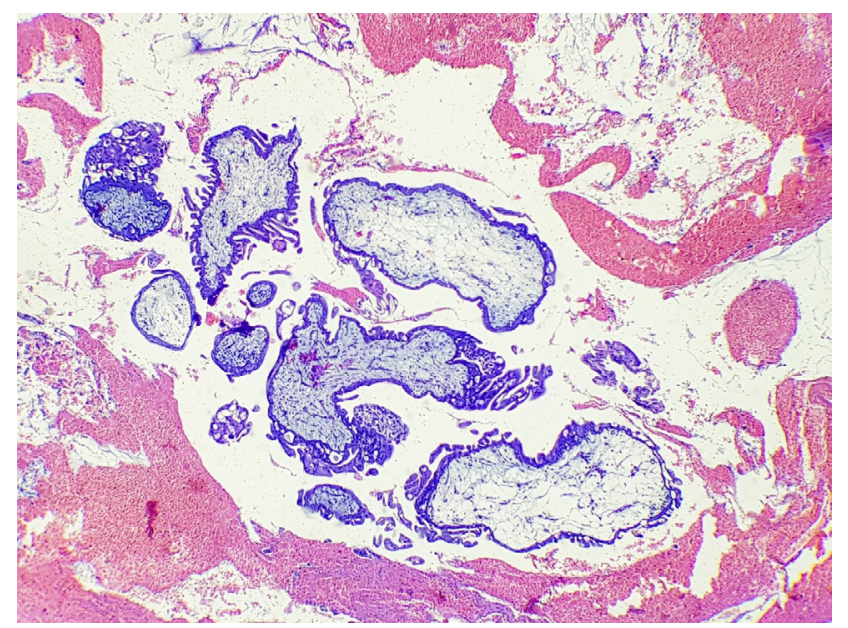


A diploidia exclui essencialmente a MHP, porém pode corresponder a $\mathrm{MHC}$ e $\mathrm{AH}$ que geralmente são diploides. A triploidia pode corresponder MHP ou um AH triploide. Por isso o dado isolado da ploidia do DNA é insuficiente para estabelecer um diagnóstico de mola hidatidiforme.

\section{CONCLUSÃO}

A concordância interobservador para o diagnóstico de DTG

\section{REFERÊNCIAS}

1. Lurain JR. Gestational trophoblastic disease I: epidemiology, pathology, clinical presentation and diagnosis of gestational trophoblastic disease, and management of hydatidiform mole. Am J Obstet Gynecol. 2010;203:539-1.

2. Braga A, Obeica B, Moraes V, Silva EP, Amim-Junior J, Rezende Filho J. Doença trofoblástica gestacional - atualização. Revista HUPE. 2014;13(3):54-60.

3. Kurman RJ, Mazur MT, Shih IM. Gestational trophoblastic tumors and related tumor-like lesions. In: Kurman RJ, Ellenson LH, Ronnet BM, editors. Blaustein's pathology of the female genital tract. 6. ed. New York: Springer; 2011. p. 1075- 1135.

4. Ellenson LH, Pirog EC. O trato genital feminino. In: Kumar V, Abbas AK, Aster JC, editors. Robbins e Cotran: Patologia - bases patológicas das doenças. 8. ed. Rio de Janeiro: Elsevier; 2010. p. 1060-71. variou de pobre a moderada. Para reduzir a variabilidade interobservador, valores de beta- HCG devem ser informados na solicitação do anatomopatológico, disponibilidade de imunocoloração para p57 e citometria de fluxo faz-se necessário nos casos dúbios e critérios padronizados devem ser observados, especialmente quando a histologia é o padrãoouro para o diagnóstico.

5. Andrade JM. Mola hidatiforme e doença trofoblástica gestacional. Rev Bras Ginecol Obstet. 2009;31(2):94-101.

6. Mecconnell TG, Murphy KM, Hafez M, Vang R, Ronnet BM. Diagnosis and subclassification of hydatidiform moles using p57 immunohistochemistry and molecular genotyping: validation and prospective analysis in routine and consultation practice settings with development of an algorithmic approach. Am J Surg Pathol. 2009;33:805-17.

7. Fukunaga M, Katabuchi H, Nagasaka T, Mikami Y, Minamiguchi $\mathrm{S}$, Lage JM. Interobserver and intraobserver variability in the diagnosis of hydatidiform mole. Am J Surg Pathol. 2005;29:942-7.

8. Crisp H, Burton JL, Stewart R, Wells M. Refining the diagnosis of hydatidiform mole: image ploidy analysis and p57KIP2 immunohistochemistry. Histopathology. 2003;43:363-73.

\section{Como citar:}

Sousa EM, Cavalcante DI, Araújo LC. Variabilidade interobservador no diagnóstico da doença trofoblástica gestacional: um estudo na residência de patologia da Universidade Federal do Ceará. Rev Med UFC. 2019 out-dez;59(4):22-26. 